\title{
Aditivo fluidificante con copolímero para mejorar la resistencia a la comprensión del hormigón
}

Flow additive with copolymer to improve compressive strength of concrete

Recibido: julio12 de 2021 | Revisado: setiembre 15 de 2021 | Aceptado: octubre 25 de 2021

\author{
Andrés Avelino Valencia Gutiérrez ${ }^{\mathrm{I}}$ \\ Carlo Magno Chávarry Vallejos ${ }^{2}$ \\ Liliana Janet Chavarría Reyes 3 \\ Joaquín Samuel Támara Rodríguez ${ }^{4}$ \\ LuIs BarRantes ManN ${ }^{5}$ \\ Darío Gonzales Roncal Araujo ${ }^{6}$ \\ Jeffrey Fernando Narrea ${ }^{7}$
}

1 Grupo de investigación de la Facultad de Ingeniería y Arquitectura . USMP, Perú

2 Grupo de Laboratorio de la Facultad de Ingeniería Civil - URL, Perú

3 Investigador de la Escuela Universitaria de Post Grado UNFV, Perú

4 Grupo de Investigación de la Universidad Ricardo Palma, Perú

Autor para correspondencia

E-mail: avalenciag@usmp.pe

\begin{abstract}
Resumen
El objetivo de esta investigación es hacer un análisis documental de investigaciones realizadas con el uso de aditivos fluidizantes, a base de copolímeros, con la finalidad de encontrar hormigones que cumplan con las exigencias del mercado. La metodología consistió en hacer un análisis documental/bibliográfica de investigaciones utilizando buscadores disponibles. Se utilizó como filtro de búsqueda el período del 2017 hasta marzo 2021, obteniendo como resultado, investigaciones que obtuvieron un Slump Flow de 32.5 $\mathrm{cm}$., para una dosis de $0.3 \%$ de aditivo, encontrándose en un rango de $0.25 \%$ a $0.40 \%$. El aditivo sintetizado en forma de peine, con codificación PCE-2, tuvo una resistencia a la compresión de $85 \mathrm{MPa}$ con adiciones minerales, ensayado a los 28 días con una relación a/c de 0.30. Para un PC-3, se obtuvo una resistencia a la compresión de $73 \mathrm{MPa}$, sin adiciones minerales, ensayado a los 28 días a una relación a/c $=0.30$. El valor promedio de resistencia a la compresión, sin adiciones minerales, fue de $61 \mathrm{MPa}$, con una relación a/c entre 0.25 a 0.30 . Se concluyó que el uso de aditivos plastificante, basados en copolímeros, tiene una influencia positiva en las propiedades del hormigón, reduciendo el agua, mejorando las resistencias altas iniciales y finales, así como la permeabilidad.
\end{abstract}

Palabras clave: Aditivo superplastificante; copolímero de injerto; ensayo de asentamiento; resistencia; cadenas laterales
Abstract
To contribute to the solution, we set the objective of making a documentary analysis of research carried out with the use of fluidizing additives, based on copolymers, in order to find concretes that meet market demands. The methodology consisted of making a documentary / bibliographic analysis of research using

(C) Los autores. Este artículo es publicado por la Revista Campus de la Facultad de Ingeniería y Arquitectura de la Universidad de San Martín de Porres. Este artículo se distribuye en los términos de la Licencia Creative Commons Atribución No-comercial - Compartir-Igual 4.0 Internacional (https://creativecommons.org/licenses/ CC-BY), que permite el uso no comercial, distribución y reproducción en cualquier medio siempre que la obra original sea debidamente citada. Para uso comercial contactar a: revistacampus@usmp.pe.

https: 
Andrés Avelino Valencia Gutiérrez - Carlo Magno Chávarry Vallejos - Liliana Janet Chavarría Reyes - Joaquín Samuel Támara Rodríguez - Luis Barrantes Mann - Darío Gonzales Roncal Araujo -

JefFrey Fernando NARREA

available search engines. The period from 2017 to March 2021 was used as a search filter, obtaining as a result, investigations that obtained a Slump Flow of $32.5 \mathrm{~cm}$., For a dose of $0.3 \%$ of additive, found in a range of $0.25 \%$ to $0.40 \%$. The synthesized comb-shaped additive, with PCE-2 coding, had a compressive strength of $85 \mathrm{MPa}$ with mineral additions, tested at 28 days with an a / c ratio of 0.30 . For a PC-3, a compressive strength of 73 MPa was obtained, without mineral additions, tested at 28 days at a ratio a / $\mathrm{c}=0.30$. The average value of compressive strength, without mineral additions, was 61 $\mathrm{MPa}$, with a w/ $\mathrm{c}$ ratio between 0.25 to 0.30 . It was concluded that the use of plasticizer additives, based on copolymers, has a positive influence on the properties of concrete, reducing water, improving initial and final high strengths, as well as permeability.

Key words: Superplasticizer additive; graft copolymer; settlement test; strength; side chains

\section{Introducción}

En los últimos años $\mathrm{y}$ debido al crecimiento del rubro de construcción, la producción de hormigón para obras de infraestructura tiene una alta demanda. Por eso, la investigación y desarrollo de nuevos aditivos superplastificantes se ha intensificado para poder no solo abastecer la demanda, sino también, para mantener la calidad y la mejora de las propiedades en estado fresco y endurecido, optimizando los tiempos en la construcción y una menor cantidad de materiales para su desarrollo.

El reductor de agua de policarboxilato (PCE) está insaturado por la copolimerización de ácido carboxílico y olefinas. Las investigaciones actuales, se centran en la influencia del agente reductor de agua sobre las propiedades mecánicas del hormigón. Entre los agentes reductores de agua tenemos, entre otros, el naftaleno y melamina Huang \& Li, (2015). Los investigadores He, Zhang, Kong, \& Wang, (2018) indican que los plastificantes PCE afectan significativamente las propiedades reológicas de las pastas de cemento porque la densidad carboxílica de PCE influye en gran medida en el rendimiento de dispersión de los superplastificante.

En ese sentido, Huang \& Yang (2018) mencionan que el hormigón autocompactante o de alta resistencia, se caracteriza por una baja relación agua-cemento y plantea desafíos para su viscosidad y capacidad de bombeo. Para ello, sintetizan un superplastificante de policarboxilato hiperramificados mediante el método de polimerización redox in situ. El superplastificante de policarboxilato PCEyel superplastificante Alifática indican Ma \& Qi (2020) que son agentes reductores de agua ampliamente utilizados en el hormigón, verificaron el comportamiento reológico de la pasta de cemento que contiene PCE y están estrechamente relacionados con la dosis de agregados.

Su \& Kong (2019) sintetizaron un superplastificante de policarboxilato (PCE) similar a un peine y copolímeros 
lineales (PAS) de ácido acrílico (AA) y ácido 2-acrilamido-2-metilpropano sulfónico (AMPS) y que impactan en la fluidez e hidratación temprana del cemento de belita de sulfoaluminato de calcio (CSAB).

Los resultados muestran que los polímeros (PAS) lineales son muy eficaces en la dispersión de pastas de cemento fresco y, también, en la hidratación temprana del cemento. Zahia, \& Karim (2018) mencionan que el efecto de la finura del cemento utilizado es muy significativo en presencia de la adición mineral y el superplastificante. El aumento de finura mejora la resistencia mecánica de los morteros ensayados $\mathrm{y}$, la adición del policarboxilato tiene un efecto beneficioso sobre el desarrollo de la resistencia mecánica, especialmente, para dosis menores o iguales al $0.8 \%$.

En las obras de infraestructura se requiere que el hormigón cumpla con la resistencia de diseño y ciertos estándares de calidad. La investigación busca identificar y conocer la influencia de los aditivos plastificante, basados en copolímeros (PCE), en los resultados de los ensayos para garantizar un adecuado control de calidad.

Chen (2012) estudia las mezclas con copolímero policarboxílico y hormigón de alto rendimiento, con aditivo compuesto, combinando la mezcla del hormigón de alto rendimiento, y concluye que mejora la trabajabilidad, la dureza, el fraguado, el endurecimiento del concreto y la calidad de resistencia.

Pan, Jiang \& Zhang (2016) indican que los superplastificantes combinados de tipo condensado y copolímero reducen la adsorción de plastificante en minerales de cemento y en la superficie de sus productos de hidratación retrasan el proceso de hidratación de C3A, y forma hidróxidos dobles en capas orgánicas, aumenta la contracción química temprana y promueve el crecimiento de la hidratación temprana del cemento.

En ese orden de ideas, Winnefeld \& Becker (2007) mostraron que el copolímero de forma de peine, con enlace éster, tiene una menor cantidad adsorbida inicial, acelerando la tasa de hidratación. Los aditivos superplastificantes, basados en copolímeros, con columna vertebral e injerto de cadenas laterales son usados por sus propiedades dispersantes a bajas dosis, excelente reducción de agua, trabajabilidad y menor contracción.

Por otro lado, Stecher \& Plank (2019), afirman que los polímeros de peine fosfatados, exhiben un rendimiento de dispersión superior a los polímeros de policarboxilatos convencionales, retardan al cemento y muestran una robustez, comparable frente a las impurezas de sulfato y arcilla.

Liu \& Lei (2015) sintetizaron una serie de plastificantes (PC) a base de policarboxilato con diferentes estructuras y estudiaron los efectos de la estructura química y las propiedades reológicas de la pasta de cemento mediante un aparato de micro electroforesis y un reómetro.

Los resultados mostraron que existe una relación entre las propiedades reológicas con la longitud de la cadena lateral y la densidad de las PC. Ibragimov \& Fediuk (2019) identificaron la influencia de los diferentes tipos de plastificantes de naftaleno, formaldehido 
y policarboxilatos durante el proceso de activación de suspensiones cementosas por el aparato de pulsación RPA. Además, indican que la incorporación de cenizas volantes en el hormigón mejora la durabilidad.

Łaźniewska-Piekarczyk (2018), indica que, la eficacia de los aditivos, con respecto al hormigón fresco y el endurecido depende de variables relacionadas con el cemento y los agregados, que se mezclan entre sí, y también, de factores externos como la temperatura ambiental. Zahia, \& Karim (2018), utilizaron ciertos aditivos orgánicos en la producción de morteros y hormigones que, influyen en la trabajabilidad y la cinética de hidratación del mortero. Esto da, como resultado, una modificación de algunas propiedades como el comportamiento reológico y la resistencia mecánica. La trabajabilidad del hormigón de alto rendimiento (HPC), según Jonbi, Arini,R., Anwar \& Ali (2018), depende del valor de asentamiento de la mezcla de hormigón. Además, la retención de asentamiento es la más sensible en comparación con un valor de asentamiento normal porque representa la durabilidad de la mezcla de hormigón.

El objetivo de la presente investigación es determinar la influencia de estos aditivos fluidizantes basados en copolímeros en el hormigón de alta resistencia a través de un análisis documental de investigaciones realizadas.

\section{Variables}

El aditivo superplastificante, basado en copolímeros, fue tratado como una variable independiente porque lo hemos estudiado por las experimentaciones y manipulaciones hechas por los diferentes autores de las investigaciones analizadas. Este aditivo se ha usado para concretos, capaces de mejorar sus propiedades mecánicas. Se emplea para conferir al hormigón fresco un mejor comportamiento en cuanto a trabajabilidad y bombeabilidad, pero también, se busca con su uso mejorar, significativamente, la resistencia y durabilidad del hormigón. Así mismo, definimos: porcentaje de aditivo superplastificante basado en copolímero, relación agua/cemento.

Las propiedades del hormigón cualitativo-nominal se han tratado como una variable dependiente por las mismas razones expuestas líneas arriba. En el estado fresco es una masa blanda y puede ser trabajada o moldeada en diferentes formas, y así, se conserva durante la colocación y la compactación. Por otra parte, después de que el hormigón ha fraguado, empieza a ganar resistencia y se endurece, conocido como estado endurecido. Hemos definido los indicadores siguientes: asentamiento del concreto de alta resistencia, resistencia a la compresión.

\section{Método}

En la presente investigación documental/bibliográfica se utilizó, como medio de información artículos, tesis, revistas, normas técnicas, libros y demás documentos. Las investigaciones revisadas utilizaron el método deductivo y de orientación aplicada porque al buscar el grado porcentual de influencia de la adición del aditivo generaron mejoras en las propiedades del hormigón. La investigación es de enfoque cuantitativo porque se utilizó información levantada 
en los ensayos de laboratorios de materiales.

Como las dos variables principales son cuantitativas, se procedió a validar la hipótesis aplicando Pearson. Luego, en base a la medición numérica y el análisis estadístico, establecimos pautas de comportamiento y conclusiones. Es así como se usaron mediciones numéricas establecidas, a través de las propiedades mecánicas y porcentajes que permitieron establecer los resultados de la investigación.

La fuente de recolección de datos fue retroelectiva, por ser información, obtenida a base de datos de estudios científicos anteriores. Es de nivel descriptivo ya que permite exponer la cantidad de aditivo superplastificante, basado en copolímeros, edades de ensayos, promedios y desviaciones para establecer los grados de influencia en las propiedades del hormigón, en estado fresco y endurecido, como también, en sus propiedades mecánicas. El diseño fue experimental, dado que se manipula deliberadamente las variables de estudio (aditivo) y al observar el comportamiento de estas se determinaron la relación de influencia entre variables y se estudia la causa (aditivo fluidificante con copolímero) para evaluar el efecto de resistencia a la comprensión del hormigón (Chavarry, Chavarría, Valencia, Pereyra, Arieta \& Rengifo 2020).

\section{Población y muestra}

La población fue estimada en base a las normas determinadas por los autores de la investigación, como, por ejemplo, la norma E-060 (2009), el número mínimo de pruebas requeridas que se establece, en no menos de 30 ensayos, para verificar los parámetros e indicadores correspondientes: Ensayo de compresión de la muestra de hormigón NTP 339.034 (2008). NTP 339.033 (2009), Práctica normalizada para la elaboración y curado de especímenes de hormigón en campo. Prueba de Slump Flow (ASTM C1611). ASTM C143- 12. Método de ensayo para el asentamiento en el hormigón de cemento hidráulico.

A continuación tenemos los criterios de inclusión para la población estudiada en la Tabla 1 y criterios de exclusión Tabla 2.

Tabla 1

Cuadro de inclusión para la población

\begin{tabular}{|cl|}
\hline Materiales & \multicolumn{1}{c|}{ Descripción } \\
\hline Agregado fino & Determinado por la NTP 400.037 \\
Agregado grueso & Huso 57 (NTP 400.037) \\
Agua & $\begin{array}{l}\text { Agua potable que cumpla con las especificaciones } \\
\text { técnicas de la Norma. }\end{array}$ \\
Aditivo & $\begin{array}{l}\text { Aditivo superplastificante basado en copolímero que } \\
\text { cumpla con las especificaciones de la norma. }\end{array}$ \\
\hline
\end{tabular}


Andrés Avelino Valencia Gutiérrez - Carlo Magno Chávarry Vallejos - Liliana Janet Chavarría Reyes - Joaquín Samuel Támara Rodríguez - Luis Barrantes Mann - Darío Gonzales Roncal Araujo JefFrey Fernando NARREA

Tabla 2

Cuadro de exclusión para la población

\begin{tabular}{cl}
\hline \multicolumn{1}{c}{ Materiales } & \multicolumn{1}{c}{ Descripción } \\
\hline Tipo de cemento & $\begin{array}{l}\text { Cemento Portland Tipo II, III, IV y V, y Cementos Adicionales (MS, } \\
\text { MH, HS, IP, ICO, IPM, etc.). }\end{array}$ \\
Agregado fino & $\begin{array}{l}\text { Todo material fino que no cumpla con las especificaciones de la (NTP } \\
\text { 400.037). }\end{array}$ \\
Agregado grueso & $\begin{array}{l}\text { Todo material grueso que no cumpla con las especificaciones Huso 57 } \\
\text { de TMN igual a 1" (NTP 400.037). }\end{array}$ \\
Agua & $\begin{array}{l}\text { Agua que no cumpla con las especificaciones de la NTP 339.088. } \\
\text { (2006) }\end{array}$ \\
Aditivo & $\begin{array}{l}\text { Aditivos acelerantes naturales o no convencionales que no se } \\
\text { encuentren normalizados o industrializados. Aditivos que cumplan } \\
\text { otras funciones }\end{array}$ \\
\hline
\end{tabular}

La Norma Técnica de Edificación E-060 hormigón armado toma de dos muestras para probetas de 6" x 12 " y, tres muestras para probetas de 4" x 8", como mínimo, por cada periodo de análisis, con la finalidad de obtener valores confiables y una dispersión aceptable. La resistencia a la comprensión se verifica según ensayos a los 3, 7, 14, 21 y 28 días (según criterios del investigador) y para el asentamiento el Slump Flow, de acuerdo con las normas: NTP 339.034 $\rightarrow$ Muestra de ensayos normalizados para la determinación de la resistencia a la compresión del hormigón, en muestras cilíndricas, y la ASTM C1611 $\rightarrow$ Ensayo de Slump Flow.

Se determinó la unidad de análisis como probetas de hormigón endurecido a diferentes días de las siguientes formas geométricas: (i) Probeta cilíndrica de 6" de diámetro por 12" y 4" x 8" de altura (NTP 339.033 y NTP 339.084). (ii) Probetas prismáticas (barras de mortero) de $15 \mathrm{~cm} \times 15 \mathrm{~cm} \times 45 \mathrm{~cm}$ (NTP 339.033).

\section{Técnicas e instrumentos de recolección de datos}

A partir de las pruebas y ensayos utilizados, por los investigadores, en cada una de las investigaciones citadas en la presente investigación se puede medir las propiedades mecánicas del concreto de las unidades de análisis en base a las normas y especificaciones.

Los instrumentos utilizados fueron formatos de ensayos para una correcta y ordenada recolección de datos basados en las especificaciones técnicas. Se realizaron los ensayos de resistencia a la compresión y ensayo de asentamiento (Slump) de las investigaciones de cada autor, documentaciones que estuvieron basadas en las normas ACI, ASTM, NTP y la europea UNE que rigen los procedimientos y materiales a utilizar para obtener diseños óptimos.

Como primer paso, empezamos con la recolección de información sobre los diferentes ensayos realizados por otros investigadores tales como: resistencia a la compresión, tracción y flexión. Con la muestra referencial, en donde se aplicaron los diferentes tipos de porcentaje de aditivos plastificante a incorporar se obtuvo el promedio aritmético con un rango de confiabilidad. Los resultados son confiables porque pertenecen a fuentes verídicas, de bases de datos aprobados 
y validados del ámbito académico y científico.

A continuación, se selecciona la información sobre los ensayos de compresión y asentamiento de las investigaciones seleccionadas. Después se realiza el análisis estadístico con un rango de confiabilidad. Se representan los datos en tablas y gráficos tipo barras verticales y horizontales en donde se aprecia el comportamiento de las variables.

\section{Resultados}

\section{Superplastificante basado en copolímeros sobre el asentamiento}

Para analizar la influencia del aditivo superplastificante basado en copolímeros sobre el asentamiento de la mezcla del hormigón, según los autores Liu \& Wang, (2015), indican que el aditivo superplastificante autosintetizado de estructura molecular denominado "multiples brazos" tuvo un asentamiento de $32.50 \mathrm{~cm}$ a una dosis de $0.30 \%$ en comparacion a un aditivo superplastificante convensional que a una dosis de $0.40 \%$ obtuvo un Slump Flow de $27.50 \mathrm{~cm}$. Ambas muestras fueron ensayadas a una relaciòn $\mathrm{a} / \mathrm{c}=0.29$. Toda informaciòn antes mencionada se proporciona (Figura 1).

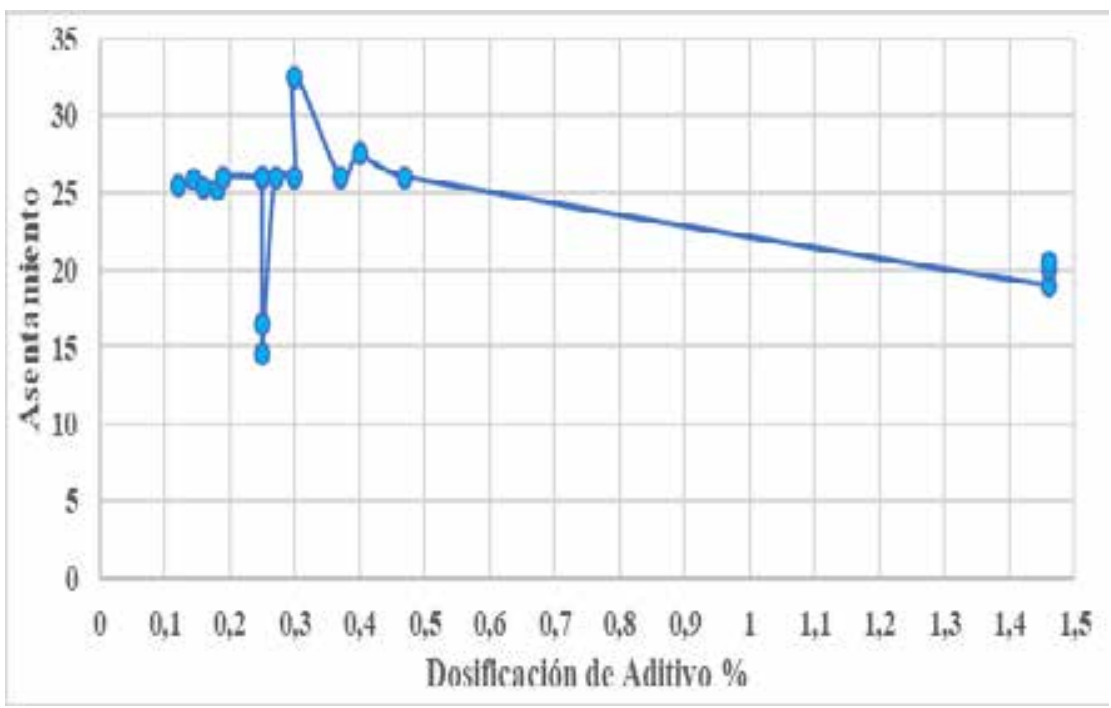

Figura 1. Asentamiento vs dosificación de aditivo (\%)

IIg \& Plank (2016) mencionan que el aditivo superplatificante sintetizado con injertos ATBS en una estructura principal de lignito tuvo Slump Flow inicial de $26.30 \mathrm{~cm}$ para la muestra Lignito (ATBS-co-AA) con una relacion molar de 1:0.15 y una dosificaciòn de $0.25 \%$ en peso de cemento en comparacion a un superplastificante convencional (BNS) que obtuvo un $25.80 \mathrm{~cm}$ para una dosificaciòn de $0.30 \%$ en peso de cemento. Para todas las muestras estudiadas por el autor se utilizó una relacion a/c constante de 0.45 .

Por otro lado, Chen (2012) indica que el aditivo superplastificante basado en copolímeros de policarboxilato P (AAco-MA) /PEG obtuvo un Slump Flow promedio máximo de $20.50 \mathrm{~cm}$ para una relación a/c=0.50 a una dosificación de $1.46 \%$ de aditivo por peso de cemento obteniendo una considerable reducción de agua. En esta investigación se utilizaron 
Andrés Avelino Valencia Gutiérrez - Carlo Magno Chávarry Vallejos - Liliana Janet Chavarría Reyes - Joaquín Samuel Támara Rodríguez - Luis Barrantes Mann - Darío Gonzales Roncal Araujo -

Jefrrey Fernando Narrea

adiciones minerales y polvo de escoria de alto horno y los investigadores Jun \& Jeong (2014) afirman que los copolímeros de policarboxilato estructuralmente modificados con injertos de cadenas laterales cortas toma como muestra el aditivo sintetizado AP25, mostró un mejor comportamiento que el hormigón patrón sin aditivos dando un Slump Flow de $16.50 \mathrm{~cm}$ con una dosificación óptima de $0.25 \%$ para una relación $a / c=0.28$. Sin embargo, el autor afirma que a pesar de que estos aditivos sintetizados presentan numerosas cadenas laterales de injerto no aumenta la fluidez de la mezcla debido a la corta longitud de estas mismas.

En el estudio de Huang \& Yang (2018), realizaron pruebas con los aditivos plastificante hiperramificados usando el método de redox in situ, se obtuvo un Slump Flow de $25.80 \mathrm{~cm}$ para una dosificación de $0.15 \%$ a una relación $\mathrm{a} / \mathrm{c}=0.29$ por encima del de la muestra patrón. Sin embargo, se requieren dosis más alta de aditivo hiperramificados para alcanzar mejores resultados que el aditivo convencional (Tabla 3).

\section{Tabla 3}

Recopilación de datos más relevantes seleccionados para el asentamiento

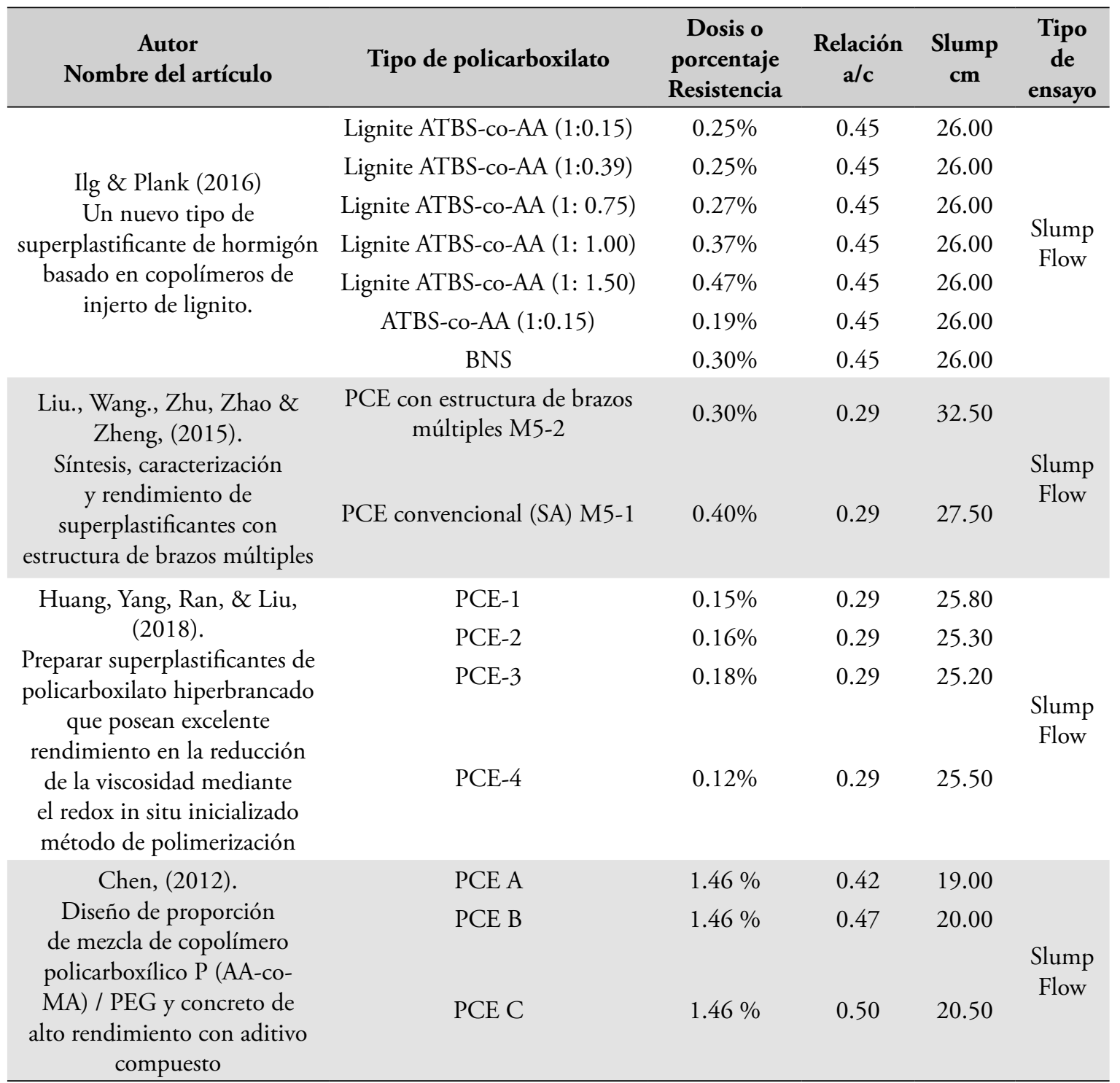




\section{Relación a/c sobre la resistencia a la compresión}

Se utilizaron aditivos de diferentes tipos de superplastificantes a base de naftaleno formaldehido y policarboxilato durante el proceso de activación de suspensiones cementosas por el aparato pulsación RPA. Se utilizó cemento Portland escoria CEM II / AS 32.5 como material aglutinante, agregado fino con una densidad aparente de $1.550 \mathrm{~g} / \mathrm{cm} 3$.agregado grueso con una densidad de $2.75 \mathrm{~g} / \mathrm{cm} 3$ y una densidad aparente de $1.40 \mathrm{~g} / \mathrm{cm} 3$. Como aditivo se utilizaron superplastificantes de diversas bases químicas (naftaleno formaldehido y policarboxilato). El uso de estos aditivos es para aumentar la resistencia inicial y las propiedades físico-mecánicas. Para los ensayos de resistencia a la compresión, se utilizaron probetas de $10 \times 10 \times 10 \mathrm{~cm}$ para todas las muestras.

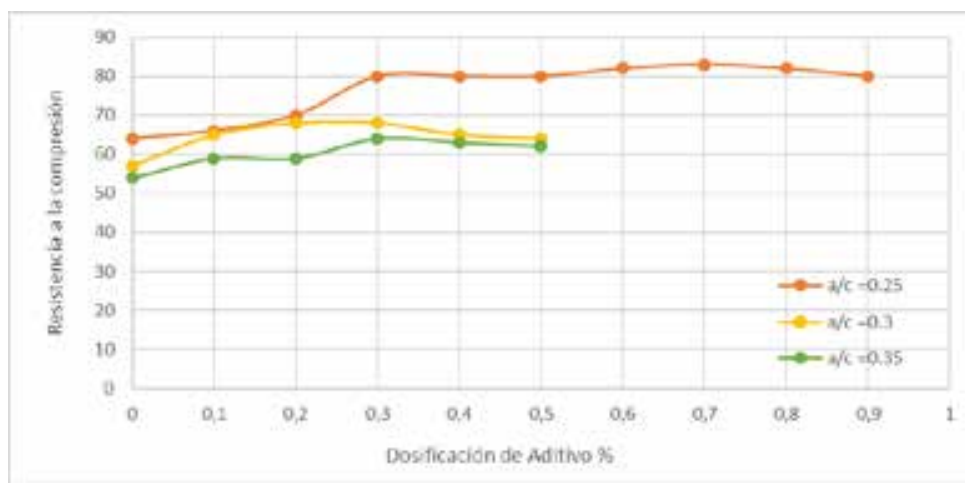

Figura 2. Resistencia a la compresión del concreto a los 28 días para diferente relación a/c.

Como se muestra en la Figura 2, se llega a una resistencia máxima de 83.00 $\mathrm{MPa}$ para una relación agua cemento a/ $\mathrm{c}=0.25$ y una dosificación de aditivo de $0.80 \%$ para el superplastificante tipo BA. Antoni \& Kusuma (2017), afirman que la dosis óptima depende del tipo de superplastificante y que la fuerza de compresion se reducirá con el uso excesivo de superplastificante. El superplastificante $\mathrm{Z}$ ayuda a la resistencia del concreto para un desarrollo rápido en el tiempo, lo que demuestra su superioridad a los otros superplastificantes. Por tanto, se puede concluir que el superplastificante $\mathrm{Z}$ promueve la reacción de hidratación en la etapa temprana, lo que refleja aún más las características del policarboxílico funcional.

Por otro lado, el efecto del PCE-2 y los materiales cementantes suplementarios sobre la resistencia a la compresión del mortero de cemento en diferentes edades. Debido a que este material cementante suplementario tenía un tamaño de partícula más pequeño que el cemento, cuyos poros podían llenarse mejor, el sistema de empaque de partículas se volvió más cerrado. Además, los materiales cementantes suplementarios tuvieron buenos efectos de forma, buena movilidad y menor adsorción de agua, por lo que podrían mejorar la trabajabilidad de la mezcla de hormigón, que mostró cierto efecto reductor de agua. Además, también encontramos que el mortero de cemento con dosis de escoria mostró una resistencia temprana más baja. Desde la síntesis de los aditivos superplastificantessss $\mathrm{o}$ adiciones minerales que afectan directamente a la resistencia a la compresión. Los datos más relevantes se aprecian en la (Tabla 4). 
Andrés Avelino Valencia Gutiérrez - Carlo Magno Chávarry Vallejos - Liliana Janet Chavarría Reyes - Joaquín Samuel Támara Rodríguez - Luis Barrantes Mann - Darío Gonzales Roncal Araujo JefFrey Fernando Narrea

Tabla 4

Tipo de aditivo con relación alc y resistencia a los 28 dias

\begin{tabular}{|c|c|c|c|c|}
\hline Autor y Título & $\begin{array}{c}\text { Tipo de } \\
\text { policarboxilato }\end{array}$ & $\begin{array}{l}\text { Aditivo } \\
\%\end{array}$ & $a / c$ & $\begin{array}{c}\text { Rc } \\
\mathrm{MPa}\end{array}$ \\
\hline $\begin{array}{l}\text { Ibragimov \& Fediuk, (2019). } \\
\text { Mejorando la resistencia inicial del }\end{array}$ & $\begin{array}{l}\text { Remicrete SP60 sin } \\
\text { activación P5 }\end{array}$ & 1.00 & 0.30 & 57.55 \\
\hline $\begin{array}{l}\text { de } \\
\text { la activación de la suspensión de cemento } \\
\text { y el uso de diversos superplastificantes }\end{array}$ & $\begin{array}{l}\text { Remicrete SP60 con } \\
\text { activación P6 }\end{array}$ & 1.00 & 0.30 & 65.72 \\
\hline \multirow{4}{*}{$\begin{array}{c}\text { Erzengin, Kaya, Özkorucuklu, Özdemir } \\
\text { \& Yıldırım (2018). } \\
\text { Las propiedades de los sistemas } \\
\text { de cemento superplastificados con } \\
\text { policarboxilatos a base de éster } \\
\text { metacrílico }\end{array}$} & PC500-1-10 & 0.30 & 0.29 & 50.00 \\
\hline & PC500-1-15 & 0.30 & 0.29 & 50.00 \\
\hline & PC950-1-10 & 0.30 & 0.29 & 55.00 \\
\hline & PC950-1-15 & 0.30 & 0.29 & 60.00 \\
\hline \multirow{11}{*}{$\begin{array}{l}\text { Antoni y Kusuma (2017) O. C., \& } \\
\text { Hardjito, D. (2017). } \\
\text { Optimización de la dosis de } \\
\text { superplastificante a base de } \\
\text { policarboxilato con diferentes tipos de } \\
\text { cemento }\end{array}$} & PMMA & 0.30 & 0.29 & 45.00 \\
\hline & S.CC & 0.60 & 0.25 & 79.00 \\
\hline & S.SV & 0.50 & 0.25 & 79.00 \\
\hline & S.BA & 0.50 & 0.25 & 83.00 \\
\hline & S.BS & 0.50 & 0.25 & 75.00 \\
\hline & S.AS & 0.50 & 0.25 & 70.00 \\
\hline & S.CC - 2 & 0.20 & 0.30 & 60.00 \\
\hline & $S . S V-2$ & 0.50 & 0.30 & 74.00 \\
\hline & S.BA -2 & 0.30 & 0.30 & 70.00 \\
\hline & S.BS -2 & 0.50 & 0.30 & 70.00 \\
\hline & S.AS -2 & 0.40 & 0.30 & 70.00 \\
\hline \multirow{4}{*}{$\begin{array}{l}\text { Raymond, Januarti, \& Triwulan. (2017). } \\
\text { Optimización del hormigón } \\
\text { autocompactante de alto rendimiento }\end{array}$} & $\begin{array}{l}\text { Humo de sílice }(\mathrm{SD})+ \\
\text { polvo de vidrio } \mathrm{R} 1\end{array}$ & 1.00 & 0.27 & 56.34 \\
\hline & $\begin{array}{l}\text { Humo de sílice }(\mathrm{SD})+ \\
\text { polvo de vidrio }(\mathrm{R} 4)\end{array}$ & $1.00 \%$ & 0.27 & 53.2 \\
\hline & $\begin{array}{l}\text { Humo de sílice }(\mathrm{SD})+ \\
\text { polvo de vidrio } \mathrm{r} 2\end{array}$ & $1.00 \%$ & 0.29 & 42.93 \\
\hline & $\begin{array}{l}\text { Humo de sílice }(\mathrm{SD})+ \\
\text { polvo de vidrio } \mathrm{R} 3\end{array}$ & $1.00 \%$ & 0.31 & 32.16 \\
\hline \multirow{2}{*}{$\begin{array}{l}\text { Raymond, Januarti, \& Triwulan. (2017). } \\
\text { Optimización del hormigón } \\
\text { autocompactante de alto rendimiento }\end{array}$} & $\begin{array}{l}\text { Humo de sílice }(\mathrm{SD})+ \\
\text { polvo de vidrio } \mathrm{R} 1\end{array}$ & $1.00 \%$ & 0.27 & 56.34 \\
\hline & $\begin{array}{c}\text { Humo de sílice }(\mathrm{SD})+ \\
\text { polvo de vidrio R3 }\end{array}$ & $1.00 \%$ & 0.31 & 32.16 \\
\hline \multirow{2}{*}{$\begin{array}{l}\text { Raymond, Januarti, \& Triwulan. (2017). } \\
\text { Optimización del hormigón } \\
\text { autocompactante de alto rendimiento }\end{array}$} & $\begin{array}{l}\text { Humo de sílice }(\mathrm{SD})+ \\
\text { polvo de vidrio } \mathrm{r} 1\end{array}$ & $1.00 \%$ & 0.27 & 56.34 \\
\hline & $\begin{array}{l}\text { Humo de sílice }(\mathrm{SD})+ \\
\text { polvo de vidrio } \mathrm{R} 3\end{array}$ & $1.00 \%$ & 0.31 & 32.16 \\
\hline \multirow[b]{2}{*}{$\begin{array}{l}\text { Xiang, Gao., \& Shi, (2020) } \\
\text { Síntesis de un nuevo policarboxilato a } \\
\text { temperatura ambiente y su influencia en } \\
\text { las propiedades de las pastas de cemento } \\
\text { con diferentes materiales cementantes } \\
\text { suplementarios }\end{array}$} & PCE- 1 & $0.15 \%$ & 0.30 & 80.00 \\
\hline & PCE-2 & $0.15 \%$ & 0.30 & 85.00 \\
\hline
\end{tabular}




\section{Relación a/c con aditivos superplastificantes basado en copolímeros}

Los autores Ibragimov \& Fediu, (2019) contrastaron que el uso de aditivo superplastificante Remicrete SP60 en las muestras por composición 5 y 6 muestran una resistencia a la compresión de $57.55 \mathrm{MPa}$ y $65.72 \mathrm{MPa}$ a los 28 días de ensayo con una dosificación de $1.00 \%$ y relación agua cemento de 0.30 para ambas composiciones, siendo superior a la muestra de composición 1 que obtuvo una resistencia a la compresión de 40.53 $\mathrm{MPa}$ a los 28 días de ensayo con una relación a/c $=0.42$ como se muestra en la (Tabla 5 y Figura 3 ).

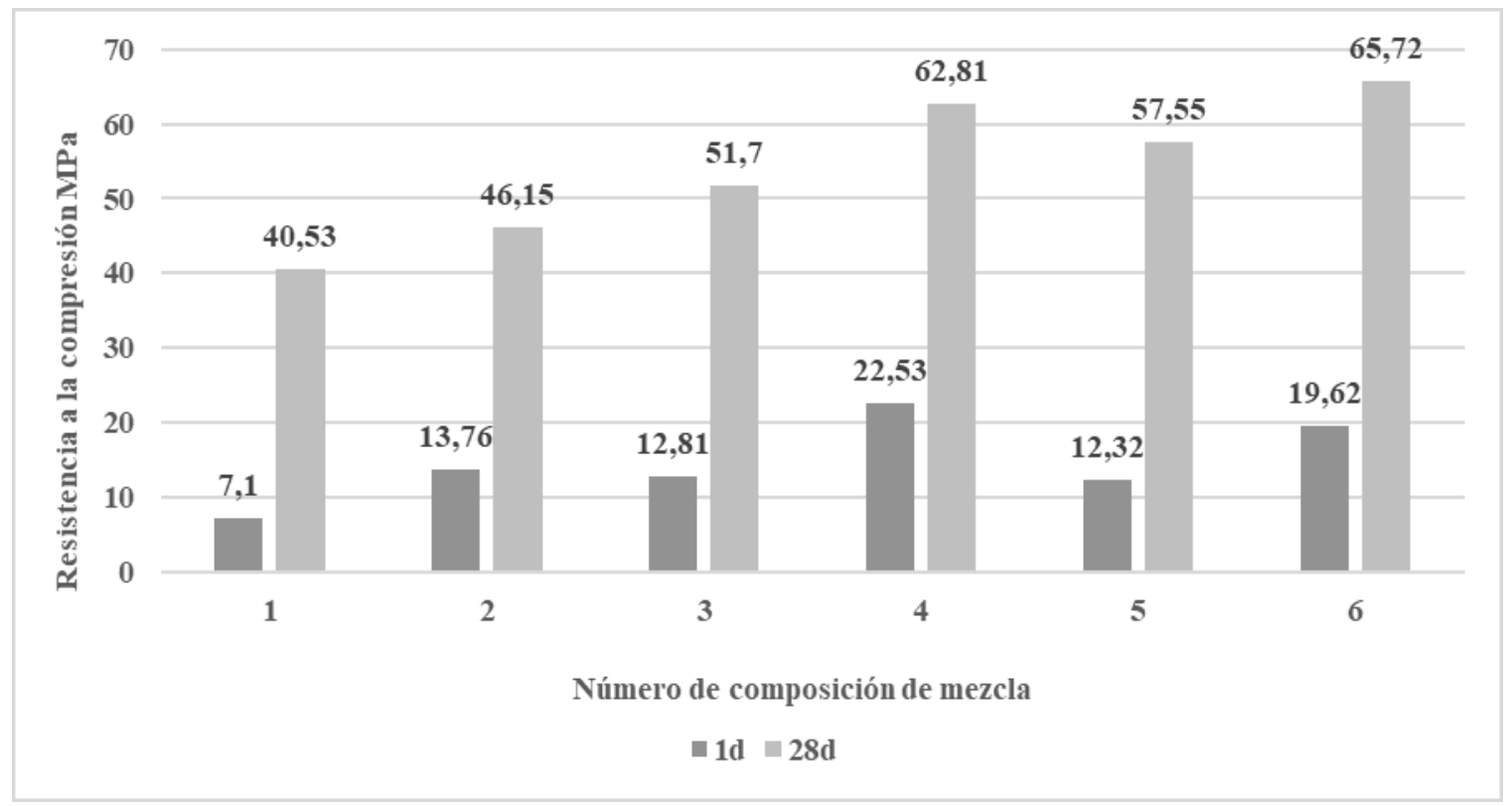

Figura 3. Resistencia a la compresión por número de composición de mezcla

Tabla 5

Propiedades fisicas el concreto y resistencia a la compresión

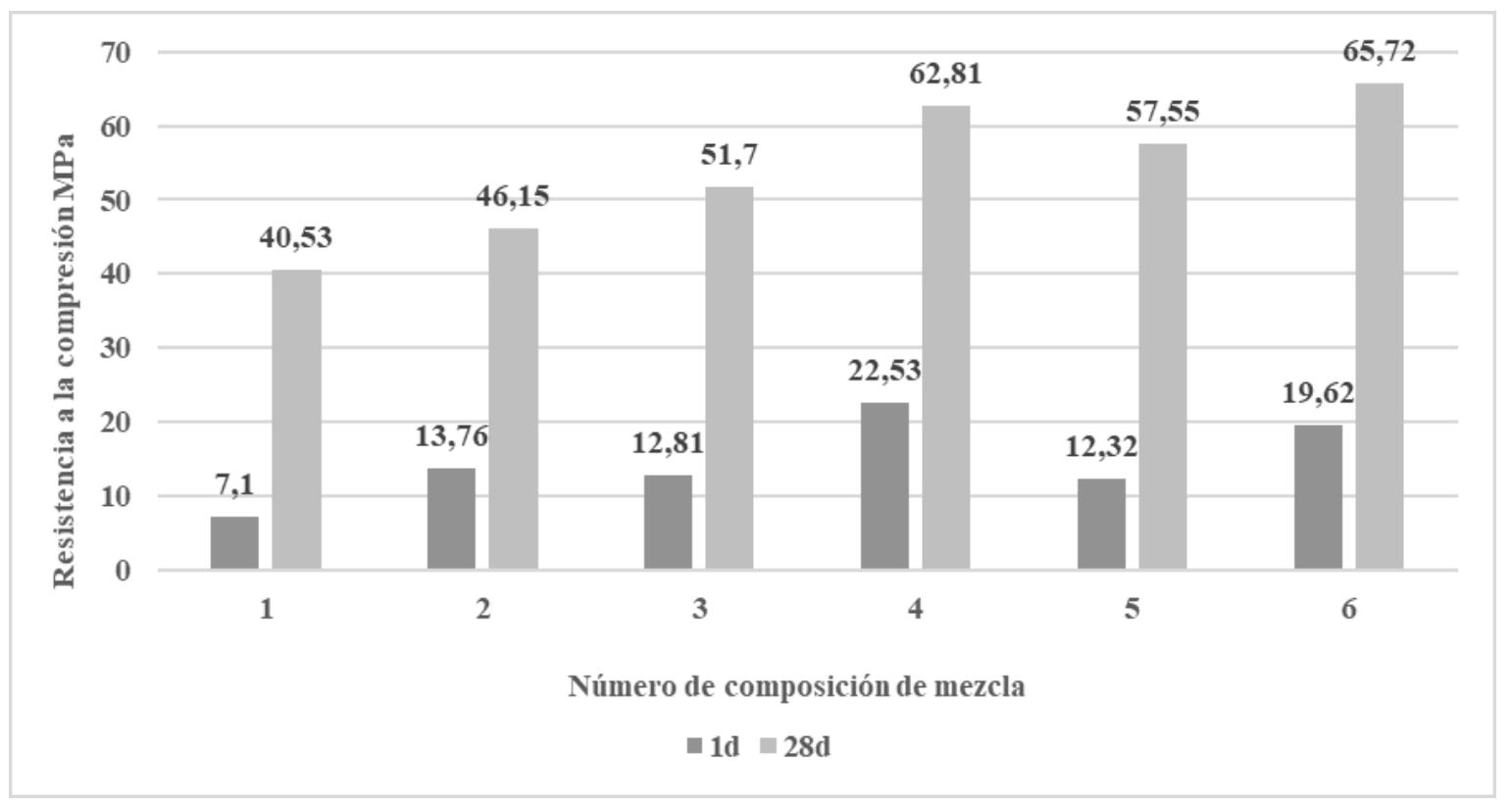


Andrés Avelino Valencia Gutiérrez - Carlo Magno Chávarry Vallejos - Liliana Janet Chavarría Reyes - Joaquín Samuel Támara Rodríguez - Luis Barrantes Mann - Darío Gonzales Roncal Araujo -

JefFrey Fernando NarRea

\section{Discusión}

Miao \& Ran (2011); Zhang, Kong, Gao \& Wang (2016); Winnefeld \& Becker (2007) y Benaicha, Hafidi Alaoui, Jalbaud, \& Burtschell (2019) indican que el aditivo superplastificante, basado en copolímero, mejora las propiedades del hormigón de alta resistencia. Los aditivos plastificantes, PCE de alto rendimiento tienen un Slump Flow promedio de $30.00 \mathrm{~cm}$ en un rango de $0.20 \%$ a $0.26 \%$ de dosificación. Para la resistencia a la compresión, se obtuvieron resultados de hasta $73.50 \mathrm{MPa}$ para una relación a/c = 0.45 .

Según los resultados de asentamiento, los autores Miao \& Ran y "Zhang, (2011), Kong, Gao, \& Wang (2016) encontraron la dosificación óptima del aditivo superplastificante basado en copolímeros mejora el asentamiento en el hormigón de alta resistencia. Dan un asentamiento promedio de $21.50 \mathrm{~cm}$ utilizando un aditivo superplastificante de copolímero anfótero con estructura tipo peine en un rango de dosificación $0.30 \%$ a 40\%. Zhang, et al., (2018), obtienen un asentamiento promedio de $30 \mathrm{~cm}$, utilizando un aditivo superplastificante PCE de alto rendimiento en una dosificación en un rango de $0.20 \%$ a $0.26 \%$. Winnefeld \& Becker (2007) y Benaicha, Hafidi, Alaoui, Jalbaud, \& Burtschell, (2019), indican que los aditivos superplastificantes basados en copolímeros mejoran la resistencia a la compresión en hormigón de alta resistencia obteniendo resultados de 54.60 MPa y 73.50 MPa respectivamente a pesar de la naturaleza del aditivo superplastificante utilizado. Sin embargo, la relación a/c fueron de 0.37 y 0.45 respectivamente.

\section{Conclusiones}

El uso de aditivo superplastificante, auto sintetizado de estructura molecular de "múltiples brazos", tuvo un Slump Flow de $32.50 \mathrm{~cm}$ para una dosis de $0.30 \%$ y se encuentra entre el rango de $0.25 \%$ a $0.40 \%$. El aditivo superplastificante sintetizado, en forma de peine con codificación PCE-2, tuvo una resistencia a la compresión de $85.00 \mathrm{MPa}$ ensayado a los 28 días con una relación a/c $=0.30$ y adiciones minerales. Para la muestra, con codificación PC-3, se obtuvo una resistencia a la compresión de $73.00 \mathrm{MPa}$ ensayado a los 28 días a una relación a/c $=0.30$, pero sin adiciones minerales.

El valor promedio de resistencia a la compresión, sin adiciones minerales, fue de 61.00 MPa con una relación a/c de 0.25 a 0.30 . El uso de aditivo superplastificante auto sintetizado, de estructura molecular denominado "múltiples brazos", tuvo un Slump Flow de $32.50 \mathrm{~cm}$ para una dosis de $0.30 \%$, en comparación a un aditivo superplastificante convencional con una dosis de $0.40 \%$ obtuvo Slump Flow de $27.50 \mathrm{~cm}$. La dosificación se encuentra entre el rango de $0.25 \%$ a $0.40 \%$ del rango optimo encontrado para la aplicación de estos aditivos.

El uso del aditivo superplastificante sintetizado, en forma de peine con codificación PCE-2, obtuvo una resistencia a la compresión de 85.00 MPa ensayado a los 28 días con una relación $\mathrm{a} / \mathrm{c}=0.30$ y adiciones minerales. Para la muestra con codificación PC-3, se obtuvo una resistencia a la compresión de $73.00 \mathrm{MPa}$ ensayado a los 28 días a igual relación $\mathrm{a} / \mathrm{c}=0.30$ pero, $\sin$ adiciones minerales. El valor promedio de resistencia a la compresión de los datos 
recopilados, fue de $61.00 \mathrm{MPa}$ actuando en un rango de relación a/c de 0.25 a
0.30, lográndose los picos más altos de resistencia.

\section{Referencias}

Antoni, J., \& Kusuma, O. (2017). Optimizing Polycarboxylate Based Superplasticizer

Dosage with Different Cement Type. Procedia Engineering: https://doi.org/10.1016/j. proeng.2017.01.442

ASTM C143-12, (2014). Método de ensayo para el asentamiento en el hormigón de cemento hidráulico.

ASTM C1611 / C1611M-18, (2018). Método de prueba estándar para el flujo de asentamiento de concreto auto consolidado, ASTM International, West Conshohocken, PA, 2018. www.astm.org

ASTM INTERNATIONAL, (2011). ASTM C143-78 Slump of Portland Cement Concrete. West Conshohocken: UNSPSC Code 30111500(Concrete and mortars).

Benaicha, M., Hafidi Alaoui, A., Jalbaud, O., \& Burtschell, Y. (2019). Dosage effect of superplasticizer on selfcompacting concrete: correlation between rheology and strength. Journal of Materials Research and Technology: https://doi. org/10.1016/j.jmrt.2019.01.015

Chavarry Vallejos, C. M., Chavarría Reyes, L. J., Valencia Gutiérrez, A. A., Pereyra Salardi, E., Arieta Padilla, J. P., \& Rengifo Salazar, C. A. (2020). Hormigón reforzado con vidrio molido para controlar grietas $y$ fisuras por contracción plástica. https:// doi.org/10.29018/issn.25881000vol4iss31.2020pp31-41

Chen, B. (2012). Mix Proportion Design of P (AA-co-MA) /PEG Polycarboxylic Copolymer and High Performance Concrete with Composite Admixture. Applied Mechanics and Materials: www. scientific.net/amm.246-247.1085

Erzengin, S., \& Kaya, K. (2018). The properties of cement systems superplasticized with methacrylic ester-based polycarboxylates. ConstructionandBuildingMaterials: https://doi.org/10.1016/j. conbuildmat.2018.01.088

He, Y., Zhang, X., Kong, Y., \& Wang, H. (2018). Influence of Polycarboxylate Superplasticizer on Rheological Behavior in Cement Paste. de Journal of Wuhan University of Technology-Mater: https://doi.org/10.1007/s11595018-1915-8

Huang, X., \& Li, X. (2015). Application of Polycarboxylate Superplasticizer in the Concrete. https://doi. org/10.1007/s11595-015-1265-8

Huang, Z., \& Yang, Y. (2018). Preparing hyperbranched polycarboxylate superplasticizers possessing excellent viscosity-reducing performance 
Andrés Avelino Valencia Gutiérrez - Carlo Magno Chávarry Vallejos - Liliana Janet Chavarría Reyes - Joaquín Samuel Támara Rodríguez - Luis Barrantes Mann - Darío Gonzales Roncal Araujo -

Jefrerey Fernando NarRea

through in situ redox initialized polymerization method. Cement and Concrete Composites: https://doi.org/10.1016/j. cemconcomp.2018.06.017

Ibragimov, R., \& Fediuk, R. (2019). Improving the early strength of concrete:Effectofmechanochemical activation of the cementitious suspension and using of various superplasticizers. Construction and Building Materials: https://doi.org/10.1016/j. conbuildmat.2019.07.313

IIg, M., \& Plank, j. (2016). A novel kind of concrete superplasticizer based on lignite. $\quad \underline{\text { https://doi.org/10.1016/j. }}$ cemconres.2015.09.004IVERSIDAD

Jonbi, J., Arini, R., Anwar, B., \& Ali Fulazzaky, M. (2018). Effect of added the polycarboxylate ether on slump retention and compressive strength of the high-performance concrete. MATEC Web of Conferences: http://dx.doi.org/10.1051/ matecconf/201819501020

Jun, Y., \& Jeong, Y. (2014). Influence of the structural modification of polycarboxylate copolymer with a low dispersing ability on the set-retarding of Portland cement. Obtenido de KSCE Journal of Civil Engineering: https://doi.org/10.1007/s12205014-1189-4

Łaźniewska-Piekarczyk, B. (2018). Influence of temperature on the properties of non-air-entrained and air-entrained cement mortars modified with different plasticisers and superplasticisers types.
MATEC Web of Conferences: https://doi.org/10.1051/ matecconf/201816304004

Liu, M., \& Lei, J. (2015). Preparation of polycarboxylate-based superplasticizer and its effects on zeta potential and rheological property of cement paste. Journal of Wuhan University of Technology-Mater: $\quad$ https://doi. org/10.1007/s11595-015-1265-8

Liu, X., \& Wang, Z. (2015). Synthesis, Characterization and Performance of Superplasticizer with a Multi-arm Structure. Obtenido de https://doi. org/10.4028/www.scientific.net/ MSF.815.594

Liu, X., Wang, Z. M., Zhu, J., Zhao, M., \& Zheng, Y. S. (2015). Synthesis, Characterization and Performance of Superplasticizer with a Multi-Arm Structure. Obtenido de Materials Science Forum: https://doi. org/10.4028/www.scientific.net/ MSF.815.594

Ma, B., \& Qi, H. (2020). Effect of aliphatic-based superplasticizer on rheological performance of cement paste plasticized by polycarboxylate superplasticizer. Construction and Building Materials: https://doi.org/10.1016/j. conbuildmat.2019.117181

Miao, C., \& Ran, Q. (2011). New Generation Amphoteric Comblike Copolymer Superplasticizer and Its Properties. Polymers and Polymer Composites: https://doi. org/10.1177/096739111101900101

Norma E.060, (2009). Concreto armado, Reglamento Nacional de 
Edificaciones. Primera Edición: Julio 2009. Biblioteca Nacional del Perú No 2009-07742.

NTP 339.033, (2009). Hormigón (concreto). Práctica normalizada para la elaboración y curado de especímenes de concreto en campo.

NTP 339.034, (2008). Hormigón (concreto). Método de ensayo normalizado para la determinación de la resistencia a la compresión del concreto, en muestras cilíndrica

NTP 339.084, (2012). Método de ensayo normalizado para la determinación de la resistencia a tracción simple del concreto, por compresión diametral de una probeta cilíndrica.

Pan, Y., Jiang, Y., \& Zhang, S. (2016). Study on the effect of condensatetype and copolymer-type combined superplasticizers on hydration of cement. Obtenido de Key Engineering Materials: https://doi. org/10.4028/www.scientific.net/ KEM.703.360

Raymond, A., Januarti, J., \& Triwulan. (2017). Optimizing High Performance Self Compacting Concrete. Obtenido de MATEC Web of Conferences: http://dx.doi.org/10.1051/ matecconf/20179701027

Stecher, J., \& Plank, J. (2019). Novel concrete superplasticizers based on phosphate esters. Cement and Concrete Research. Cement and Concrete Research: https://doi.org/10.1016/j. cemconres.2019.01.006
Su, T., \& kong, X. (2019). Effects of comblike PCE and linear copolymers on workability and early hydration of a calcium sulfoaluminate belite cement. Obtenido de Cement and Concrete Research: https://doi.org/10.1016/j. cemconres.2019.105801

Winnefeld, F., \& Becker, S. (2007). Effects of the molecular architecture of comb-shaped superplasticizers on their performance in cementitious systems. Obtenido de Cement and Concrete Composites: https://doi.org/10.1016/j. cemconcomp.2006.12.006

Xiang, S., \& Gao, Y. (2020). Synthesis of Pectiniform Polyurethane-Modified Polycarboxylate and Its Preliminary Application in UltrahighPerformance Concrete. Advances in Civil Engineering: http://dx.doi. org/10.1155/2020/8859093

Zahia, D., \& \& Karim, E. (2018). Effect ofcement fineness and polycarboxylate dosage on the rheological and mechanical behavior of a mortar. MATEC Web of Conferences: http://dx.doi.org/10.1051/ matecconf/201814901037

Zhang, Y., Kong, X., Gao, L., \& Wang, J. (2016). Rheological behaviors offresh cement pastes with polycarboxylate superplasticizer. Journal of Wuhan University of TechnologyMater: https://doi.org/10.3390/ app10103496 